\title{
Interaction structures in the psychodynamic therapy of a boy diagnosed with Asperger's Disorder: a single-case study
}

\author{
Vera Regina Rohnelt Ramires ${ }^{1}$, Cibele Carvalho ${ }^{1}$, Fernanda Munhoz Driemeier Schmidt ${ }^{1}$, \\ Guilherme Pacheco Fiorini ${ }^{1}$, \& Geoff Goodman ${ }^{2}$
}

\begin{abstract}
It is important to investigate the outcomes of psychotherapy and, especially, its process. Regarding child psychodynamic psychotherapy, available studies are in smaller numbers. Therefore, we still do not fully know the mechanisms of change in treatments with this age group. The Child Psychotherapy Q-Set (CPQ) was designed to analyze the therapeutic process with children. It permits one to identify interaction structures (i.e., repetitive patterns of interaction) and how they change in the course of a treatment. Based on these assumptions, the aim of this study was to identify and to analyze the interaction structures in the psychodynamic therapy of a boy diagnosed with Asperger's disorder and possible changes in his psychic organization. A mixed longitudinal study, based on the Systematic Case Study procedure, was performed. Approximately 30 months of the boy's psychotherapy were analyzed using the CPQ. The Rorschach method was used as the outcome measure. Four interaction structures were identified, using the CPQ: Active, confident and lively child, competing with connected, mentalizing and accepting therapist; Withdrawn and defensive child with uncertain, unresponsive and didactic therapist; Accepting therapist with demanding, provocative and hostile child; and Reassuring, supportive, nondirective therapist with a compliant and not spontaneous child. Two interaction structures varied over time. Some changes in Rorschach variables were detected after two years of treatment.
\end{abstract}

Keywords: child psychotherapy process; interaction structures; case study

This study focuses on the interaction structures in the psychodynamic therapy of a boy diagnosed with Asperger's disorder and possible changes in his psychic organization. Psychodynamic psychotherapy is derived from a set of basic principles which were originated in psychoanalysis, related to the concepts of dynamic unconscious, transference, countertransference and resistance (Gabbard, 2007). This approach aims to address focal situations, identify and resolve developmental conflicts, facilitate the

\footnotetext{
${ }^{1}$ Universidade do Vale do Rio dos Sinos, UNISINOS, Brazil ${ }^{2}$ Long Island University, United States of America

Correspondig Author: Vera Regina Rohnelt Ramires, Universidade do Vale do Rio dos Sinos, UNISINOS, Brazil; email to: verareginaramires@gmail.com
}

acquisition of insight regarding the unconscious motivations of behaviors related to the principal conflicts, and help the patient resume a normal course of development (Kernberg, 2006). The most essential aspect of child psychodynamic treatment is interpreting the patient's conflicting impulses and defenses. This technique allows the ego to function more adaptively, thereby enabling the modification of defenses, a change in the superego and a greater instinctual gratification. Over the course of psychotherapy, it is hoped that the child's regressive tendencies will decrease; they will overcome inhibitions and developmental blocks; and released and neutralized energy will become available for sublimated activity.

For many years, psychodynamic psychotherapy was considered lacking a basis of scientific evidence and 
was not on the lists of empirically supported treatments (Midgley \& Kennedy, 2011). In the field of psychodynamic treatment of adults this situation began to change in recent years, with the publication of a number of important reviews and meta-analyzes (De Matt, Jonghe, Schoevers \& Dekker, 2009; Gerber et al., 2011; Leichsenring, 2005; Leichsenring \& Rabung, 2008; Leichsenring, Rabung, \& Leibing, 2004; Rabung \& Leichsenring, 2012; Shedler, 2012; Town, Diener, Abbass, Leichsenring, Driesse, \& Rabung, 2012). Based on Randomized Controlled Trials (RCT) or not, these revisions showed that psychodynamic psychotherapy is at least equal to other forms of treatment regarded as evidence-based and that patients receiving this treatment not only keep the gains after its end, but often continue to improve.

On the other hand, research examining the efficacy and effectiveness of child psychodynamic psychotherapy lagged behind, although some recent publications have been showing a growing interest in this field (Deakin \& Nunes, 2008; 2009; Goodman \& Athey-Lloyd, 2011; Kennedy \& Midgley, 2007; Midgley \& Kennedy, 2011; Midgley, Anderson, Grainger, Vuckovic-Nesic, \& Urwin, 2009; Palmer, Nascimento \& Fonagy, 2013). In addition, although there is an increase of studies on child psychodynamic psychotherapy, indicating an evidence base on the effectiveness and efficacy of this psychotherapeutic modality, the need for more research in this field remains, especially considering that the therapeutic process is still poorly understood. Analyzing why and how changes occur as a result of a therapeutic intervention (i.e., the nature of therapeutic action) has the potential to help identifying the active ingredients or change mechanisms that underlie a successful clinical intervention (Fonagy, 2003; Kazdin, 2009; Midgley, 2007).

Asperger's disorder and Psychodynamic Psychotherapy. Asperger's disorder was added to the DSM-IV (American Psychiatric Association, 2000) in 1994, and it shares many characteristics of autism: significant impairment in social interaction, and the presence of restricted, repetitive and stereotyped behavior (DSM-IV; APA, 2000). On the other hand, severe cognitive and language delays found in patients with autism are not present.

The DSM-5 (American Psychiatric Association, 2013) has included the Asperger's disorder in the group of Autism Spectrum Disorder. It is necessary to specify "With or without accompanying intellectual impairment", and "With or without accompanying language impairment", as well the level of support required, which informs the severity of the diagnosis. In this study, we decided to keep the DSM-IV description, considering it more clear and appropriate for this case study.

The treatment for a child with Asperger's disorder may include behavior management, behavioral parent interventions, curriculum adaptation, vocational training, and assistive technology (Goodman \& Athey-Lloyd, 2011). These treatment approaches favor structured interventions that focus on behavior. The lack of social - cognitive functioning is addressed by teaching those skills through various cognitive-behavioral therapy strategies. However, the inner organization of these children must be understood. For many psychoanalytic approaches, social behavior is developed through relationships that involve emotionally meaningful exchanges. Besides, children who present with Asperger's disorder have a greater capability for theory of mind than do children with autism, being able to make use of displacement in psychodynamic play-based approaches.

Based on these assumptions, psychodynamic therapists continue to apply this approach in the treatment of the disorder, reporting good results (Bromfield, 2000; Fonseca, 2009; Goodman \& Athey-Lloyd, 2011; Olesker, 1999; Pozzi, 2003; Shuttleworth, 1999; Topel \& Lachmann, 2008). Shuttleworth (1999) has suggested that children with Asperger's disorder share some similarities with adults with narcissistic personality disorder and can be subjected to psychoanalytic intervention. Fonseca (2009) hypothesised that in autistic states there is a distortion in the construction of a dialogic space. Such a space, in which self and other define each other mutually, would be the final result of early dyadic transactions. The author reported the potential of psychodynamic treatment describing the psychotherapy of a 12-year-old-boy in which the development of a dialogic space was the locus of negotiation of otherness/selfness.

Nevertheless, this work has not been yet subjected to empirical study (Goodman \& Athey-Lloyd, 2011). It is important to develop empirical investigation which addresses this topic.

\section{Interaction Structures and Therapeutic Action.}

Regarding the nature of the therapeutic action, one can consider two main lines of thought: the mutative effect of interpretation and the interpersonal interaction. In the first case, the emphasis is on patient self-knowledge and insight. Interactive models, on the other hand, emphasize interpersonal and relationship factors as empathy, sense of security, continuity, the holding environment and the therapeutic alliance (Ablon \& Jones, 2005; Jones, 2000; Luyten, Blatt \& Mayes, 2012).

Luyten, Blatt and Mayes (2012) hypothesize the centrality of the therapeutic relationship in treatment, conceptualizing the therapeutic change process as a series of compatible and incompatible experiences in this relationship, and frustration and gratification both inside and outside the therapeutic relationship. For the authors, the therapeutic relationship can be seen not only as the vehicle of change, but should also be considered to contain poten- 
tial iatrogenic effects.

It was in this context that Jones (2000) developed the concept of interaction structures (IS), which can be positive or negative (Ablon \& Jones, 2005). Consequently, for the authors, it is necessary to study the dyad to understand the therapeutic process and its results.

According to Jones (2000, p. xv), interaction structures are "repeated, mutually influencing interactions between analyst and patient that are a fundamental aspect of therapeutic action". IS have been studied successfully in treatments with adults (Ablon \& Jones, 2005; Goodman, Edwards \& Chung, 2014; Jones, 2000) and with children (Goodman \& Athey-Lloyd, 2011; Schneider, Midgley \& Duncan, 2010).

Jones (2000), in a pioneering study in this approach, identified the IS in the psychotherapy of three adult patients diagnosed with Major Depressive Disorder and determined how they could be linked to therapeutic change in patients. In the first and in the second case, three interpretable clusters (or IS) resulted from factor analysis: "Collaborative Exploration", "Ambivalence / Compliance", and "Provoking Rescue" in the first case; "Collaborative Exploration," "Resistant and Withdrawn" and "Angry Interaction" in the second. Regarding the third case, four IS were formulated: "Therapist Neutral Acceptance", "Therapist Suppresses Patient's Negative Self Representations", " Psychodynamic Technique ", and " Patient Dysphoric Affect"

There was a clinically significant improvement in the first and in the third cases. In the second case, the symptoms fluctuated considerably during treatment. There was some improvement in the end, but at the time of follow-up assessment after one year, the symptoms returned to pretreatment levels (Jones, 2000). Jones concluded that IS may play a mutative role in different ways for different patients. He considered it unlikely that a specific therapist's action (interpretation of transference or supportive interventions) means the same thing for all patients. The subjective meaning of the observable processes varies for each dyad. The recognition, interpretation and understanding of the significance of interaction patterns are important components of the change process.

The pioneering work of Jones was succeeded by other studies in the field of research process in psychodynamic psychotherapy, based on Q methodology (Goodman \& Athey-Lloyd, 2011; Goodman et al., 2014; Schneider, Midgley \& Duncan, 2010; Schneider, Pruetzel-Thomas \& Midgley, 2009). Also with adults, Goodman et al. (2014) sought to identify the IS that characterized the treatment of five patients in crisis with borderline personality disorder. These patients participated in six months of three times per week psychodynamic psychotherapy, and completed an outcome measure (Symptom
Checklist-90-Revised [SCL-90-R]) every week. One hundred twenty-seven sessions were coded using the PQS. Four IS were identified: "Collaborative Relationship with Supportive, Reassuring Therapist", "Therapist Empathic Attunement", "Erotized Therapeutic Relationship" and "Directive Therapist with Compliant Patient". The magnitude of these IS varied within and between dyads over time.

Goodman et al. (2014) also found that the correlations of IS over time were inversely proportional to the correlations of these structures with patient's psychological distress levels. In addition, the IS " Therapist Empathic Attunement " was correlated with two different results in two cases, one of them positive and the other one negative.

Two studies identified IS in child psychodynamic psychotherapy, based on the Child Psychotherapy QSet (CPQ), a procedure equivalent to the PQS, which aims to analyze the psychotherapy process between children and therapists. Schneider, Midgley, and Duncan (2010) reported the IS identified in the psychotherapy of Helen, aged 11, and the results of this treatment. Helen was described at the beginning of treatment as highly isolated, with low self-esteem and difficulties in concentration. At that time, she met diagnostic criteria for Major Depressive Disorder, Generalized Anxiety Disorder, Panic Disorder and Avoidant Disorder of Childhood according to the DSM-IV-TR (American Psychiatric Association, 2000). Helen participated in psychoanalysis with a woman analyst for three years, with four weekly sessions. Her treatment was successful, which was also confirmed by two outcome measures (the childreported Child Depression Inventory [CDI], and the mother-reported Child Behavior Checklist [CBCL]).

In order to explore the therapeutic process over time, Schneider et al. (2010) used the CPQ. They analyzed nine sessions (three at the beginning, three in the middle, and three at the end of treatment) coded by two independent judges in random order. Factor analysis was conducted to identify clusters of items that account for the most important variations in the process. A principal components analysis with varimax rotation produced a solution of three factors, which explained $51 \%$ of the variance among the CPQ items. The IS identified were "Bringing Out the Withdrawn Child", "Working with States of Anxiety and Resistance", and "Coming Out of the Shell". The authors identified a composite of interpretive and supportive elements in the analytical work with Helen. It was not clear the extent to which the transference relationship was a focus of this work. One should point out that these results should be taken with caution, given the small number of sessions analyzed with the CPQ.

Finally, the study developed by Goodman and Athey-Lloyd (2011) also examined the IS in child psychodynamic psychotherapy, in this case between a child with Asperger's Disorder and two therapists. 
Using the CPQ, the authors analyzed two years of psychotherapy of a boy aged 6, each year with a different therapist. Four IS were identified. They differed between the two therapists and also over time within each dyad.

The four IS identified by Goodman and AtheyLloyd (2011) were "Reassuring, Supportive, Nondirective Therapist with a Compliant, Curious Child Building Insight and Positive Feelings", "Helpful, Mentalising, Confidente Therapist with Expressive, Comfortable, Help-Seeking Child", "Judgemental, Misattuned Therapist with Distant, Emotionally Disconnected, Misanderstood Child", and "Accepting Therapist with Playful, Competitive Child". The authors found, for instance, that the third factor became more characteristic for Therapist 1 over the year (this therapist conducted the first year of psychotherapy): $r=.41, p$ $<.05$. Moreover, the first factor became less characteristic for this therapist throughout the year: $r=-.56$, $p<.01$. They also found that the overall magnitude of the third factor was significantly higher for Therapist 2 than for Therapist 1: $t(51)=-2.72, p<.01$. The first and fourth factors became less characteristic during the second year of treatment: $r=-.45, p<.05$, and $r=$ $-.65, p<.001$, respectively. These results confirm the hypothesis that IS fluctuate over time within treatments and between the two therapists treating the same child. Based on these findings, the authors concluded that the therapist makes an independent contribution to the psychotherapy process, when the patient remains constant.

The reviewed studies make clear the importance of research on the therapeutic process, specifically on the vicissitudes of IS, establishing links with the results, both within and outside sessions. Furthermore, change assessments in psychotherapy have been focused almost exclusively on the measure of symptomatic improvement. However, according to the psychodynamic approach, an important goal of psychotherapy is to promote changes in the psychological structure - long-term symptomatic relief accompanied by lasting changes in patient mental functioning (Jones, 2000; Luyten, Blatt, \& Mayes, 2012). Based on these assumptions, the aim of this study was to identify and to analyze the interaction structures in the psychodynamic therapy of a boy diagnosed with Asperger's disorder and possible changes in his psychic organization. Our hypotheses were the following: 1) It would be possible to identify the IS in the psychotherapy of a boy diagnosed with Asperger's disorder, using the CPQ; 2) The IS would vary over time; and 3) It would be possible to identify some positive changes in patient's psychic organization.

\section{Method}

\section{Participants}

The participants were a boy called Peter (a pseudo- nym) and his therapist. Peter was eight years old at the onset of psychotherapy, and his parents sought help because he did not perform school assignments and exhibited symptoms of anxiety. Peter's therapist was female; she was at the beginning of her work as therapist. She held a master's degree in clinical psychology. Her theoretical orientation was based on psychodynamic psychotherapy. Her supervisor was female, had training in psychoanalytic psychotherapy and about 30 years of clinical experience.

\section{Research Design}

The present study used a longitudinal, descriptive, repeated-measures design based on the systematic case study (SCS) method (Edwards, 2007). The SCS method is a specific research method that aims to analyze the therapeutic process. It is characterized by its idiographic (the intensive study of a single case), longitudinal, and intensive approaches (as it uses repeated measures, enabling the client's response to the various components of an intervention to be examined closely). It may represent an extension of clinical practice and employ several mechanisms to achieve methodological rigor and control bias (Eells, 2007).

The SCS method can use either normal clinical practice or formal research activity as source of data (Edwards, 2007). It can be used to build and refine clinical theory, to develop and refine models for clinical practice, and to achieve evidence for effectiveness and efficaccy, according to the author.

\section{Measures}

Child Psychotherapy Q-Set (CPQ) - The CPQ (Schneider \& Jones, 2006, 2012) is used to analyze the psychotherapeutic process among 3- to 13-year-olds and was applied in the current study. This instrument consists of 100 items, each containing a statement that describes a relevant feature of the treatment process corresponding to a) the child's attitudes (i.e., feelings, behaviors, or experience); b) the therapist's actions and attitudes; and c) the nature of the patienttherapist interaction. The 100 statements were selected from a set of items chosen from a wide-ranging review of studies on child psychotherapy that included empirically validated methods and psychoanalytic approaches (Schneider, 2003; Schneider, PruetzelThomas, \& Midgley, 2009). To improve the instrument's reliability, a coding manual provides clear definitions and examples of each item, with descriptions of behaviors that might be identified from videos of sessions. The instrument is applied to the video of the psychotherapy session. Each statement receives a score ranging from 1 to 9 , according to how much it is more or less characteristic of the session, or neutral. A fixed number of items must be placed in each category resulting in a normal distribution. 
Rorschach: The Rorschach method was used to analyze potential changes during the psychotherapy. It was applied, coded, and interpreted based on Exner's (2003) Comprehensive System (CS). Weiner (2000) reports studies that show that raters have established substantial interrater agreement on all variables coded by Exner's CS. Moreover, the reliability of data in the Rorschach Structural Summary has been documented in many test-retest studies with children and adults, with test-retest intervals ranging from 7 days to 3 years. Primary Rorschach variables reached stability coefficients between 0.70 and 0.80 according to Weiner.

The Rorschach CS provides detailed reports for each of its variables coded, collected from hundreds of adults and children in clinical and nonclinical samples, providing more standardized information about personality processes than most other investigative personality instruments (Weiner, 2000). It is useful for the exploration of the structure and dynamics of personality. This method has been used in many studies, in order to analyze changes during the therapeutic process and after termination (Bram, 2010; Exner \& Andronikof-Sanglade, 1992; Gronnerod, 2004; Heedea et al., 2009; Nascimento, 2001; Weiner, 2004; Weiner \& Exner, 1991; Yazigi et al., 2013). These studies report the use of the Rorschach method before and after psychotherapy, in follow-up assessments, and during the process while monitoring the treatment.

Considering the difficulties exhibited by Peter, we analyzed the following four Rorschach clusters: self-perception, interpersonal relationship, affect modulation, and openness to experience.

\section{Procedure}

Following the parents' request for treatment, their child was assessed to confirm the need for psychotherapy. All ethical recommendations were followed. The psychotherapy was based on the psychodynamic approach. The child's sessions lasted 50 minutes and were first scheduled on a weekly basis, and later two times/week (after 82 sessions). Monthly interviews were conducted with the child's parents to collect additional data and orient them to the psychotherapeutic process. All the child's sessions were video-recorded.

To analyze the sessions, six psychologists with clinical experience were trained to use the CPQ coding system. The interrater reliability index achieved by the examiners was greater than an intraclass correlation of 0.7 for at least 10 training sessions.

Two raters were randomly assigned to and independently evaluated each session. The videos were coded in random order. Five of the six raters were blind to the patient's history and diagnosis. We analyzed the sessions that occurred in the first 30 months of treatment, as this is an ongoing psychothera- py. Fifty-four sessions (from the 120 during the 30 months of treatment) were randomly selected for analysis (about one every two sessions).

After watching the therapy session videos, the raters arranged the items into nine groups ranging from the least (category 1 ) to the most (category 9) characteristic items for a particular session. To establish interrater reliability, intraclass correlation coefficients were calculated. Each session received a final score from the average of judges ratings. The global average was calculated. The interrater reliability varied from .58 to $.82(m=.71)$ in Peter's sessions. SPSS 22.0 was used for this purpose.

The composited scores of the 54 psychotherapy sessions were submitted to a principal components factor analysis with varimax rotation. Pearson correlations were used to analyze changes in the resulting interaction structures over time.

A psychologist trained in the use of the Rorschach CS administered this test prior to the onset of psychotherapy as well as at 12 and 24 months. On all three occasions, rapport building in the presence of the Peter's therapist preceded the actual application of the test.

Two raters with specialized training coded and interpreted the Rorschach protocols. The interrater reliability for each protocol varied from .81 to .93 $(m=.86)$. Ribeiro, Semer, and Yazigi's (2012) normative data were used for comparison.

\section{The case description and assessment}

Peter was eight years old when his parents sought psychotherapeutic care for him. They were concerned with their son's performance at school. They received many complaints because he did not perform school assignments and exhibited symptoms of anxiety. Peter's parents did not perceive other difficulties in their son. Peter's parents had separated recently, and his symptoms appeared during this time.

The initial assessment was based on interviews with the parents, the boy and on CBCL (answered by the mother, the father and Peter's teacher). The House-Tree-Person Test was also used (Buck, 2003), as well the Child Depression Inventory.

The sessions revealed a withdrawn, self-restrained boy who spoke little and in a quiet voice. As a rule, he always played the same game in his sessions and was distant from his feelings. He presented good cognive capacities. The initial assessment led to a diagnosis of Asperger's disorder'.

\section{Results}

Table 1 shows the 10 most and the 10 least characteristic CPQ items during the 30 months of Peter's psychotherapy.

A specific focus or theme was very characteristic of Peter's sessions (item 23) as, in general, he always 
wanted to play the same game with the therapist (item 64). However, his play did not show spontaneity (item 95) nor a symbolic quality (item 71 , uncharacteristic). He did not engage in makebelieve play (item 71). Peter was distant from his feelings (item 56) and unwilling to examine thoughts, reactions, or motivations related to his problems (item 58). He did not seek for intimacy with the therapist (item 10). He was competitive with her (item 39).

Table 1. 10 most and 10 least characteristic CPQ items of Peter's psychotherapy

\begin{tabular}{cc} 
CPQ Item & Score (mean) \\
\hline
\end{tabular}

Goodman et al., 2014). The following IS were identified:

IS 1: Active, Confident and Lively Child, Competing with Connected, Mentalizing and Accepting Therapist (Cronbach's $\alpha=.91$ ): This IS was characterized by the connection between therapist and child. Humor was present. Peter was confident, lively, and competing with the therapist. Peter was connected to his feelings, including aggressive ones. The therapist was accepting and connected to Peter. She displayed a mentalizing stance.

This IS became more characteristic during the 30 months of treatment $(r=.63, p<.01)$. Figure 1 shows IS 1 over time.

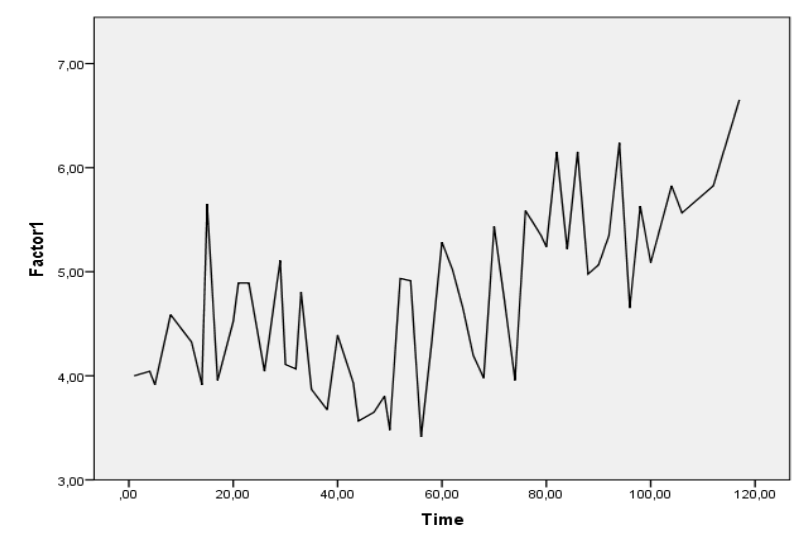

Figure 1. IS 1 over Time

IS 2: Withdrawn and Defensive Child with Uncertain, Unresponsive and Didactic Therapist (Cronbach's $\alpha=.92$ ): In this IS we observed a withdrawn, dependent, defensive, insecure and inhibited child. The aggression was directed toward himself. The therapist was unresponsive, distant, disconnected, uncertain. She did not make interventions. There was a sustained focus on Peter's play, even though it was repetitive and stereotyped.

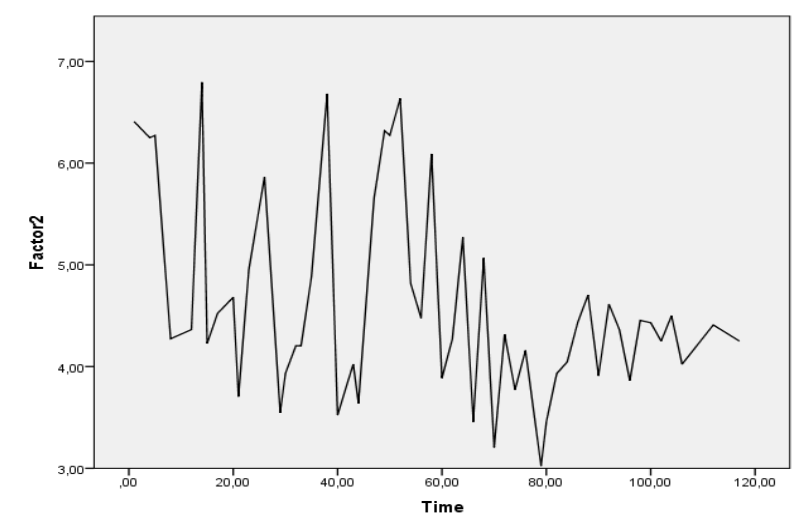

Figure 2. IS 2 over Time

This IS became less characteristic during the 30 months of treatment $(r=-.38, p<.01)$. Figure 2 shows IS 2 over time.

IS 3: Accepting Therapist with Demanding, Provo- 
cative and Hostile Child (Cronbach's $\alpha=.84$ ): A negative transference possibly was present in this IS. Peter was demanding, provocative, hostile, making important use of projective defense mechanisms. The therapist's stance was characterized by a calm and silent observation, and acceptance without interpreting unconscious contents.

IS 4: Reassuring, Supportive, Nondirective Therapist with a Compliant and Unspontaneous Child (Cronbach's $\alpha=.87$ ): In this IS Peter communicated without affect and spontaneity, was compliant, and interacted with a reassuring, supportive and flexible therapist, who did not interpret unconscious contents, defenses, and child's mood changes.

Interaction structures 3 and 4 did not change over time. Table 2 shows the items in each IS and the factor loading:

Table 2. Child Psychotherapy Q-Set (CPQ) Item Factor Loadings for the Four Interaction Structures

\begin{tabular}{cc}
\hline CPQ Item & $\begin{array}{c}\text { Factor } \\
\text { loading }\end{array}$ \\
\hline
\end{tabular}

IS 1: Active, Confident and Lively Child,

Competing with Connected, Mentalizing and Accepting Therapist $(\alpha=.91)$

$72-C$ is active.

44 - C feels wary or suspicious [vs. trusting and secure].

94 - C feels sad or depressed [vs. cheerful and joyous].

$13-\mathrm{C}$ is animated or excited.

39 - $\mathrm{C}$ is competitive, rivals with $\mathrm{T}$.

7 - $\mathrm{C}$ is anxious and tense [vs. calm and relaxed].

58 - $\mathrm{C}$ appears unwilling to examine thoughts, reactions, or motivations related to problems.

74 - Humor is used.

59 - C feels inadequate and inferior [vs. effective and superior].

56 - $\mathrm{C}$ is distant from his or her feelings.

84 - $\mathrm{C}$ expresses anger or aggressive feelings.

38 - $\mathrm{T}$ and $\mathrm{C}$ demonstrate a shared vocabulary or understanding when referring to events or feelings.

57 - $\mathrm{T}$ attempts to modify distortions in C's beliefs.

64 - C draws T into play.

81 - T emphasizes feelings to help C experience them more deeply.

41 - $\mathrm{C}$ does not feel understood by $\mathrm{T}$.

73 - C expresses fears or displays phobic behavior.

32 - $\mathrm{C}$ achieves a new understanding or insight.

33 - C expresses feelings about needing someone or being close to someone.

99 - $\mathrm{T}$ offers help or guidance.

70 - C struggles to control feelings or impulses.

42 - C ignores or rejects T's comments and observations.

0,74

0,72
12 - T models unspoken or unelaborated emotions.

IS 2: Withdrawn and Defensive Child with Uncertain, Unresponsive and Didactic Therapist

$$
(\alpha=.92)
$$

$18-\mathrm{T}$ is judgmental and conveys lack of acceptance.

3 - T's remarks are aimed at encouraging C's speech.

31 - $\mathrm{T}$ asks for more information or elaboration.

82 - T helps $\mathrm{C}$ manage feelings.

$9-\mathrm{T}$ is nonresponsive [vs. affectively engaged].

65 - T clarifies, restates, or rephrases C's communication.

29 - The quality of C's play is fluid, absorbed [vs. fragmented, sporadic].

61 - C feels shy and embarrassed [vs. unselfconscious and assured].

86 - $\mathrm{T}$ is confident, self-assured [vs. uncertain or unsure].

37 - $T$ behaves in a didactic manner.

88 - Material of the hour is meaningful and relevant to $C$ 's conflicts.

52 - T makes explicit statements about the end of the hour, upcoming weekend, or holiday.

24 - T's emotional conflicts intrude into the rela-

tionship

0,53

0,52

85 - C's aggression is directed toward self.

28 - $T$ accurately perceives the therapeutic process.

80 - C behaves in a dependent fashion [vs. insists on independence].

19 - C asks for advice or information.

25 - $\mathrm{C}$ has difficulty leaving the session.

17 - $T$ actively exerts control over the interaction (e.g., structuring, introducing new topics).

75 - Interruptions, breaks in the treatment, or termination of therapy are discussed.

10 - C seeks greater intimacy with the $T$.

69 - C's current or recent life situation is emphasized.
IS 3: Accepting Therapist with Demanding, Provocative and Hostile Child $(\alpha=.839)$

5 - C has difficulty understanding T's comments.

$51-\mathrm{C}$ attributes own characteristics or feelings to T.

53 - C conveys awareness of own internal difficulties.

34 - C blames others, or external forces, for difficulties.

20 - $\mathrm{C}$ is provocative; challenges $\mathrm{T}$ or rules and boundaries of the therapy hour.

49 - C conveys or expresses mixed or conflicted feelings about $\mathrm{T}$. 
1 - C expresses negative feelings (e.g., criticism, hostility) toward T [vs. expresses approval or admiration].

76 - T makes links between C's feelings and experience.

100 - T draws connections between the therapeutic relationship and other relationships.

90 - C's dreams or fantasies are discussed.

35 - C's self-image is a theme.

IS 4: Reassuring, Supportive, Nondirective

Therapist with a Compliant and Unspontaneous Child $(\alpha=.870)$
$66-\mathrm{T}$ is directly reassuring.

62 - T points out a recurrent theme in C's experience or conduct.

40 - $\mathrm{C}$ communicates without affect.

78 - $\mathrm{C}$ is compliant.

50 - $\mathrm{T}$ draws attention to feelings regarded by $\mathrm{C}$ as unacceptable (e.g., anger, envy, or excitement).

47 - When the interaction with $\mathrm{C}$ is difficult, $\mathrm{T}$ accommodates $\mathrm{C}$.

67 - T interprets warded-off or unconscious wishes, feelings, or ideas.

79 - T comments on changes in C's mood or affect.

98 - The therapy relationship is discussed.

$54-\mathrm{C}$ is clear and organized in verbal expression.

95 - C's play lacks spontaneity.

27 - There is a focus on helping $\mathrm{C}$ plan behavior outside the session.

Note: $\mathrm{C}=$ child and $\mathrm{T}=$ therapist

We also analyzed possible changes that occurred during the 30 months of psychotherapy, using the Rorschach method. The four clusters that were analyzed, described in Table 3,included: openness to experience, affect modulation, self-perception and interpersonal relationship. These variables describe important difficulties one typically observes in a person diagnosed with Asperger's disorder. Besides, these four clusters were recommended by Exner and Andronikof-Sanglade (1992) and Nygren (2004) to assess change in psychotherapy and were related to the difficulties presented by Peter.

When Peter came to psychotherapy, he was a withdrawn and resistant boy. He tended to perceptual simplification and extensive use of intellectual control (according to the number of responses and Lambda index). He also tended toward egocentrism, and showed a reduced interest in the external world (elevated egocentrism index). Responses related to human content showed a distorted view of himself and social environment as well as difficulties with identification processes.
Peter was cautious and reserved and presented immature and regressive postures in relationships (according to the CDI and responses of human content [Hd]). His Rorschach protocol indicated an impaired capacity to establish affective bonds. Confirming the results of other variables, regarding affect modulation, Peter had difficulties identifying and expressing his emotions, which made him emotionally and socially withdrawn.

After one year of treatment, the Rorschach protocol was quite intriguing. Even if his production capacity improved $(\mathrm{R}=18)$, the Lambda index was 0 , which means that all his answers had the Form (F) as determinant (characteristic of a poor protocol or a resistant respondent). He showed a reduction in the index of relational deficit. At the same time, the egocentrism index increased, although the responses of human content and responses of parahuman content and partial perceptions were more balanced. There was a slight increase of interpersonal interest (pure $\mathrm{H})$. The results regarding affect modulation were poor: he showed an increased level of tension as well as constriction and emotional blocking. He was using rigid defensive efforts.

At the end of two years of treatment, an improvement in his focus of attention and openness to experience were observed (number of responses and decrease of Lamba index). The egocentrism index was within normal limits for Peter's age, although his protocol still showed low self-esteem and identification with partial objects (responses of human content, pure $\mathrm{H}$ and $\mathrm{Hd}$ ). His tendency to establish superficial relationships and act distant was still present. However, Peter began to identify and express emotions as these variables were within normal limits for his age (Afr, SumC', WsumC, FC, CF, C).

\section{Discussion}

Children who present with Asperger's disorder usually have similar characteristics: significant impairment in social interaction, repetitive and stereotyped patterns of behavior, interests or activities, and absence of severe cognitive and language delays (APA, 2000; Goodman \& Athey-Lloyd, 2011). According to Pozzi (2003), they are not aware of other people's feelings, and their minds can be closed, impenetrable, and refractory.

Analyzing the average of the 10 most and the 10 least characteristic CPQ items of Peter's psychotherapy (see Table 1), we can observe the above characteristics. Peter was not a spontaneous child, and he engaged in very poor interactions with the therapist. He always played the same game in his sessions in silence. He liked to win this game and was angry if he lost. When that happened, he used to go to the bathroom and stay there for 10,15 or even 20 minutes. He did not answer the therapist's attempts to approach him in these moments. Therefore, interventions like interpretation and work on the use of de- 
Table 3. Rorschach Results at Three Points of the Peter's Psychotherapy

\begin{tabular}{|c|c|c|c|}
\hline \multirow[t]{2}{*}{ Rorschach clusters } & \multicolumn{3}{|c|}{ Protocols } \\
\hline & Beginning & 12 months & 24 months \\
\hline \multicolumn{4}{|l|}{ Openness to experience } \\
\hline Responses (production capacity) & $14 \downarrow$ & 18 & 23 \\
\hline Lambda index & $6 \uparrow$ & 0 & 1,55 \\
\hline \multicolumn{4}{|l|}{ Self-perception } \\
\hline $3 r+(2) / R$ (egocentrism index $)$ & $0,43 \uparrow$ & $0,5 \uparrow$ & 0,13 \\
\hline $\begin{array}{l}\mathrm{H}:(\mathrm{H})+\mathrm{Hd}+(\mathrm{Hd}) \text { (ratio between responses of human content } \\
\text { and responses of parahuman content and partial perceptions) }\end{array}$ & $1: 5 \uparrow$ & 02:03 & $1: 5 \uparrow$ \\
\hline \multicolumn{4}{|l|}{ Interpersonal relationships } \\
\hline CDI (index of relational deficit) & $5 \uparrow$ & 3 & 3 \\
\hline Human content (total responses of human content) & $6 \uparrow$ & 5 & $6 \uparrow$ \\
\hline Pure $\mathrm{H}$ (responses of human content) & 1 & 2 & 1 \\
\hline Hd (incomplete human form) & $5 \uparrow$ & $3 \uparrow$ & $5 \uparrow$ \\
\hline \multicolumn{4}{|l|}{ Affect modulation } \\
\hline Afr (ability to modulate affects) & $0,55 \uparrow$ & $0,38 \downarrow$ & 0,44 \\
\hline $\begin{array}{l}\text { SumC': WSumC (relation between the degree of affective } \\
\text { constriction and processing of emotions) }\end{array}$ & 01:01 & 00:00 & 03:03 \\
\hline $\mathrm{FC}: \mathrm{CF}+\mathrm{C}$ & 00:01 & 00:00 & 01:02 \\
\hline
\end{tabular}

Note: $\uparrow$ increased result compared with the mean and standard deviation of the age group; $\downarrow$ decreased result compared with the mean and standard deviation of the age group

fenses were not among the most characteristic items during the analyzed period.

The four IS identified in Peter's psychotherapy reflect such dynamics. Three IS seem more related to a positive transference-countertransference matrix (IS 1,3 and 4), while IS 2 seems more related to a negative one. The IS 2 depicted a withdrawn and defensive child, insecure and inhibited, who did not express his aggression. In turn, the therapist was unresponsive and disconnected, and did not intervene. Repetitive and stereotyped play was present, and the interaction between Peter and the therapist was distant. The items in Table 2 illustrate this IS and we wonder how much it reflects Peter's psychopathology or the therapist's characteristics and actions in these moments (her personality, countertransferece, knowledge and experience).

As we can observe in Figure 2, this IS was more prominent during the first 15 months of treatment (until session 60). This period coincided with poor outcomes according to the Rorschach method (about the end of one year of treatment). In this period, assessing the progress of psychotherapy, the therapist and her supervisor decided to adopt interventions that sought to promote the child's mentalizing capacity, helping Peter to identify and express his feelings. Thus, the therapist should replace a more neutral stance for an interaction in which the feelings and emotions would be emphasized to help Peter to experience them. We also can observe in Figure 2 that this IS became less characteristic after session 60 .

On the other hand, IS 1 became more characteristic during this same period. This IS showed more connection between the therapist and Peter.
The therapist displayed a mentalizing stance and was accepting and connected, while Peter was confident, lively and competing with the therapist, with a sense of humor. Figure 1 shows a significant increase of this IS over time.

In contrast with IS 1 and 2, regarding IS 3 and 4 we did not observe change over time. In IS 3 (Accepting Therapist with Demanding, Provocative and Hostile Child), we hypothesized the presence of a negative transference, because Peter was provocative, hostile and probably making important use of projective defense mechanisms. Notwithstanding, the therapist's stance was marked by acceptance, support, without making use of interpretation of unconscious contents.

Similarly, IS 4 was characterized by a reassuring, supportive and nondirective therapist with a compliant and unspontaneous child. This IS was very similar with an IS identified by Goodman and Athey-Lloyd (2011), in a study with a similar boy diagnosed with Asperger's disorder. Nine items in our IS 4 were found in a similar IS in their study.

Goodman and Athey-Lloyd (2011) identified four IS, three of them representing a positive transferencecountertransferece matrix, as in the present study. They also concluded that the therapist influenced the therapist-patient interaction structures, and that these patterns waxed and waned over time during the course of treatment. Their IS 3 Judgemental, Misattuned Therapist with Distant, Emotionally Disconnected, Misanderstood Child bears some resemblance with our IS 2, Withdrawn and Defensive Child with Uncertain, Unresponsive and Didactic Therapist".

Thus, our hypotheses one and two were supported in this study. The CPQ proved to be very sensitive to 
the psychotherapy process, and it permitted us to identify the IS of this treatment. Hypothesis 2 was partially supported to the extent that IS 1 and 2 were found to vary over time. Jones (2000) highlighted that recognition, interpretation and understanding of the significance of interaction patterns are important components of the therapeutic process and its changes.

In the same vein, Luyten et al. (2012), who consider the therapeutic change process as a series of compatibility and incompatibility experiences in the therapeutic relationship, had discussed also the possibility of interaction structures' potential iatrogenic effects. Such statements can help us to understand IS in Peter's treatment.

From the author's perspective, the treatment process should be understood as "a series of unfolding interactions, both at conscious and unconscious levels, between two individuals, with moments of experienced compatibilities and uncompatibilities, moments of meeting, understanding, and mutuality versus moments of separation and misunderstanding" (Luyten et al., 2012, p. 353).

The fact that IS 3 and 4 did not change over time may mean that some IS are supposed to be more constant in psychodynamic psychotherapy. These IS may express a necessary repetitive pattern: a demanding and hostile child with an accepting and supportive therapist. These interaction patterns may reflect the difficulties of children with Asperger's disorder and their impairment in social interaction as well as the repetitive and stereotyped patterns of behavior and interests. Their difficulties identifying and expressing emotions may help to explain the distant interaction between Peter and his therapist, Peter's lack of spontaneity, his distance from his feelings, his compliance, and his communication without affect, present in items that make up the IS 2 and 4 (see Table 2).

Shuttleworth (1999) and Pozzi (2003) have reported on the psychoanalytic psychotherapy of two boys, aged 10 and 12 , respectively, both diagnosed with Asperger's disorder. They described the big challenges they faced during the therapeutic process of these boys and the enormous difficulties they posed to their therapists. The traditional psychoanalytic technique was not suitable, and working on transference interpretation, defenses and so on did not produce any effect. Pozzi reported a variation on an infant-observation technique used to reach and to communicate with her patient, helping him to articulate some sense of himself and his feelings.

In this sense, we observed that supportive elements and viewing the therapist as a new object, as discussed in Schneider et al. (2010), were important features in Peter's psychotherapy reflected in IS 1, 3 and 4. Interventions aimed at enhancing mentalization seemed to promote changes in IS and in some variables detected by the Rorschach method. These interventions were present in IS 1 (see Table 2). Interestingly, Goodman and Athey-Lloyd (2011) also observed this kind of intervention in IS 2 of their study "Helpful, Mentalizing, Confidente Therapist with Expressive, Comfortable, Help-Seeking Child". Perhaps these kinds of interventions can be useful with patients diagnosed with Asperger's disorder, like Peter.

We observed that Hypothesis 3 was partially supported. Changes in some Rorschach variables were identified. However, these changes were not linear, in contrast with IS 1 and 2. Luyten et al. (2012) have discussed that the changes in psychotherapy are not linear, and that some interaction structures may have iatrogenic effects (if they are not understood and/or interpreted). Furthermore, the characteristics of Asperger's disorder sets limits for treatment. In the evaluation performed at 12 months of treatment we observed worsening in some Rorschach variables (egocentrism index, level of tension, emotional blocking). On the other hand, at 24 months, many variables were within normal limits for Peter's age. Peter began to identify and express emotions, although he still showed low self-esteem and a tendency to establish superficial relationships. Despite his difficulties, Peter showed a positive attachment to his therapist. For these reasons, he has continued in psychotherapy.

\section{Conclusion and Future Directions}

The study of IS is an important tool to analyze the psychotherapeutic process, providing many lessons and insights. The Q-methodology is suitable and very sensitive to this endeavor.

Some limitations of the present study should be pointed out. The use of outcome measures was limited in frequency, what did not permit some statistical analysis that would be able to relate these measures to the IS.We did not analyze the statistical significance of the changes in Rorschach variables because we had only three data points. These limitations could be overcome in future research.

Considering the findings of this study and the reviewed literature, we would suggest that different interaction structures could be identified in different patients' treatment with different pathologies. Each dyad is unique, but at the same time it seems that in the treatment of patients with the same diagnosis we typically find some similarity in patterns of interaction in the therapist-patient dyad. This finding can be useful to clinicians and help in identifying and understanding repetitive patterns of interaction.

According to this study, for a child diagnosed with Asperger's disorder, it is important to have an accepting, connected, reassuring, and supportive therapist. Furthermore, a mentalizing stance seems to have the potential to promote change within and outside the therapeutic process.

We suggest that future studies could analyze in- 
teraction structures in the treatment of children with different ages, different pathologies, and different theoretical approaches. Moreover, the intensive use of outcome measures, linked to the process measures, may bring light to the psychodynamic psychotherapy process with children who present Asperger's disorder.

\section{Notes}

${ }^{1}$ According to DSM-5, Peter's diagnosis would be Autism Spectrum Disorder, level 1 (without concomitant intellectual commitment and without impairment of language, APA, 2013).

\section{Acnowledgment}

Supporting Agencies: National Council for Scientific and Technological Development, CNPq, Brazil.

\section{References}

Ablon, S. J., \& Jones, E. (2005). On analytic process. Journal of American Psychoanalytic Association, 53(2), 541-568.

American Psychiatric Association. (2000). Diagnostic and statistical manual of mental disorders. ( $4^{\text {th }}$ ed.) Waschington, DC: Author.

American Psychiatric Association. (2013). Diagnostic and statistical manual of mental disorders. Fifth Edition. Waschington, DC: Author.

Bram, A. D. (2010). The relevance of the Rorschach and patient-examiner relationship in Treatment Planning and Outcome Assessment. Journal of Personality Assessment, 92(2), 91-115.

Bromfield, R. (2000). 'It's the tortoise's race: long-term psychodynamic psychotherapy with a high functioning autistic adolescente". Psychoanalytic Inquiry, 20(5), 732-745.

Buck, J. (2003). H - T - P: casa-árvore-pessoa. São Paulo: Vetor.

De Matt, S., Jonghe, F., Schoevers, R., \& Dekker, J. (2009). The effectiveness of long-term psychoanalytic therapy: A systematic review of empirical studies. Harvard Review of Psychiatry, 17(1), 1-23.

Deakin, E. K., \& Nunes, M. L. T. (2009). Effectiveness of child psychoanalytic psychotherapy in a clinical outpatient setting. Journal of Child Psychotherapy, 35(3), 290-301.

Edwards, D. J. A. (2007). Collaborative versus adversarial stances in scientific discourse: Implications for the role of systematic case studies in the development of evidencebased practice in psychotherapy. Pragmatic Case Studies in Psychotherapy, 3 (1), 6-34.

Eells, T. D. (2007). Generating and generalizing knowledge about psychotherapy from pragmatic case studies. Pragmatic Case Studies in Psychotherapy, 3 (1), 35 - 54.

Exner, J. E., \& Andronikof-Sanglade, A. (1992). Rorschach changes following brief and short-term therapy. Journal of Personality Assessment, 59(1), 59-71.

Fonseca, V. R. J. R. M. (2009). The autistic dialogic style: a case of Aperger's Syndrome. Journal of Child Psychotherapy. 35(3), 250-261.

Fonagy, P. (2003). The research agenda: The vital need for empirical research in child psychotherapy. Journal of Child Psychotherapy, 29(2), 129-136.

Gabbard, G. (2007). Principais modalidades: psicanalítica/psicodinâmica. In G. O. Gabbard, J. S. Beck, \& J.
Holmes (Eds.). Compêndio de Psicoterapia de Oxford (pp. 1429). Porto Alegre, Brasil: Artmed.

Gerber, A., Kocsis, J., Milrod, B., Roose, S., Barber, J., Thase, M., Perkins, P., \& Leon, A. (2011). A quality-based review of randomized controlled trials of psychodynamic psychotherapy. American Journal of Psychiatry, 168, 19-28.

Goodman, G., \& Athey-Lloyd, L. (2011). Interaction structures between a child and two therapists in the psychodynamic treatment of a child with Asperger's disorder. Journal of Child Psychotherapy, 37(3), 311-326.

Goodman, G., Edwards, K., \& Chung, H. (2014). Interaction structures formed in the psychodynamic therapy of five patients with borderline personality disorder in crisis. Psychology and Psychotherapy: Theory, Research and Practice, 87, 15-31.

Gronnerod, C. (2004). Rorschach assessment of changes following psychotherapy: a meta-analytic review. Journal of Personality Assessment, 83(3), 256-276.

Heedea, T., Rungea, H., Storebøa, O. J., Rowleyb E., \& Hansen, K. G. (2009). Psychodynamic milieu-therapy and changes in personality - what is the connection? Journal of Child Psychotherapy, 35(3), 276-289.

Jacobson, N. S., \& Truax, P. (1991). Clinical significance: A statistical approach to defining meaningful change in psychotherapy research. Journal of Consulting and Clinical Psychology, 41, 51-65.

Jones, E. (2000). Therapeutic action. A guide to psychoanalytic therapy. London: Jason Aronson Inc.

Kazdin, A. E. (2009). Understanding how and why psychotherapy leads to change. Psychotherapy Research, 19(4-5), 418-428.

Kennedy, E., \& Midgley, N. (2007). Process and outcome research in child, adolescent and parent-infant psychotherapy: a thematic review. London: North Central London Strategic Health Authority.

Kernberg, O. (2006). A psicanálise, a psicoterapia psicanalítica e a psicoterapia de apoio: controvérsias contemporâneas. In Agressividade, narcisismo e autodestrutividade na relação psicoterapêutica (pp. 117-144). Lisboa: Climepsi.

Leichsenring, F. (2005). Are psychodynamic and psychoanalytic therapies effective? A review of empirical data. International Journal of Psychoanalysis, 86, 841-68.

Leichsenring, F., \& Rabung, S. (2008). The effectiveness of long-term psychodynamic psychotherapy: a metaanalysis. Journal of the American Medical Association, 300, 1551-1565.

Leichsenring, F., Rabung, S., \& Leibing, E. (2004). The efficacy of short-term psychodynamic psychotherapy in specific psychiatric disorders: a meta-analysis. Archives of General Psychiatry, 61, 1208-16.

Luyten, P., Blatt, S. J., \& Mayes, L. C. (2012). Process and outcome in psychoanalytic psychotherapy research: The need for a (relatively) new paradigm. In R. A. Levy, J. S. Ablon, \& H. Kächele (Eds). Psychodynamic psychotherapy research: evidence-based practice and practice-based evidence. (pp 345-359). New York: Human Press.

Midgley, N. (2007). Researching the process of psychoanalytic child psychotherapy. In E. Kennedy, \& N. Midgley. Process and outcome research in child, adolescent and parentinfant psychotherapy: a thematic review (p. 8-53). London: North Central London Strategic Health Authoriry.

Midgley, N., Anderson, J., Grainger, E., Vuckovic-Nesic, T., \& Urwin, C. (Ed.). (2009). Child psychotherapy and research: New approaches, emerging findings. NewYork, NY: Routledge.

Midgley, N., \& Kennedy, E. (2011). Psychodynamic psychotherapy for children and adolescents: a critical review of the evidence base. Journal of Child Psychotherapy, 37(3), 
232-260.

Nascimento, S. R. G. F. (2001). The contributions of the Rorschach method to the field of Psychotherapy. Psicologia. Teoria e Prática, I(1), 85-92.

Nygren, M. (2004). Rorschach Comprehensive System variables in relation to assessing dynamic capacity and ego strength for psychodynamic psychotherapy. Journal of Personality Assessment, 83(3), 277-292.

Olesker, W. (1999). Treatment of a boy with atypical ego development. The Psychoanalytic Study of the Child. 54, 25-46.

Palmer, R., Nascimento, L. N., \& Fonagy, P. (2013). The state of the evidence base for psychodynamic psychotherapy for children and adolescents. Child and Adolescent Psychiatric Clinics of North America, 22, 149-214.

dx.doi.org/10.1016/j.chc.2012.12.001

Pozzi, M. E. (2003). The use of observation in the psychoanalytic treatment of a 12-year-old boy with Asperger's syndrome. International Journal of Psycho Analysis, 84, 1333-1349.

Rabung, S., \& Leichsenring, F. (2012). Effectivenes of long-term psychodynamic psychotherapy: first metaanalytic evidence and its discussion. In R. A. Levy, J. S. Ablon, \& H. Kächele (Eds). Psychodynamic psychotherapy research: evidence-based practice and practice-based evidence. (pp. 27-50). New York: Human Press.

Schneider, C. (2003). The development of the Child Psychotherapy Q-set. Doctoral Dissertation. University of California, Berkeley.

Schneider, C., \& Jones, E. E. (2006). Child Psychotherapy Q-Set. Coding Manual. Berkeley: University of California. Unpublished Manual.

Schneider, C., \& Jones, E. E. (2012). Appendix IB. Child Psychotherapy Q-Set. Coding Manual. In R. A. Levy, J. S. Ablon, \& H. Kächele (Eds). Psychodynamic psychotherapy research: evidence-based practice and practice-based evidence. (pp 611-626). New York: Human Press.

Schneider, C., Midgley, N., \& Duncan, A. (2010). A "motion portrait" of a psychodynamic treatment of an 11-yearold girl: Exploring interrelations of psychotherapy process and outcome using the Child Psychotherapy Q-Set. Journal of Infant, Child and Adolescent Psychotherapy, 9, 94-107.
Schneider, C., Pruetzel-Thoma, A. \& Midgley, N. (2009). Discovering new ways os seeing and speaking about psychotherapy process: The Child Psychotherapy Q-Set. In N. Midgley, J. Anderson, E. Grainger, T. Vuckovic-Nesic, \& C. Urwin (Eds.). Child psychotherapy and research: new approaches, emerging findings (pp. 72-84). New York, NY: Routledge.

Shedler, J. (2012). The efficacy of psychodynamic psychotherapy. In R. A. Levy, J. S. Ablon, \& H. Kächele (Eds). Psychodynamic Psychotherapy Research: Evidence-Based Practice and Practice-Based Evidence (pp. 9-26). New York: Humana Press.

Shuttleworth, J. (1999). The suffering of Asperger children and the challenge they presente to psychoanalytic thinking. Journal of Child Psychotherapy, 25(2), 239-265.

Topel, E-M. \& Lachmann, F. M. (2008). Life begins on an ant farm for two patients with Asperger's syndrome. Psychoanalytic Psychology, 25(4), 602-617.

Town, J. M., Diener, M. J., Abbass, A., Leichsenring, F., Driesse, E., \& Rabung, S. (2012). A meta-analysis of psychodynamic psychotherapy outcomes: evaluating the effects of research-specific procedures. Psychotherapy, 49(3), 276-290.

Weiner, I. B. (2000). Princípios da interpretação do Rorschach (M. C. V. M. Silva, trad.). São Paulo: Casa do Psicólogo.

Weiner, I. B. (2004). Monitoring psychotherapy with performance-based measures of personality functioning. Journal of Personality Assessment, 83(3), 323-331.

Weiner, I. B. \& Exner, J. E. (1991). Rorschach changes in long-term and short-term psychotherapy. Journal of Personality Assessment, 53(3), 453-465.

Yazigi, L., Areco, K. C. N., Semer, N. L., Fiore, M. L. M., Barros, R., Lerman, T. G., Abela, R. K., Avancine, C. O., \& Marques, T. C. (2013). Psychotherapy evaluation by Rorschach performance assessment system. Fragmentos de Cultura, 23(4), 512-526.

Submitted: July 2015 Accepted: November 2015 Published: December 2015 